\title{
X-ray emission from Saturn
}

\author{
J.-U. Ness ${ }^{1}$, J. H. M. M. Schmitt ${ }^{1}$, S. J. Wolk ${ }^{2}$, K. Dennerl ${ }^{3}$, and V. Burwitz ${ }^{3}$
}

\author{
${ }^{1}$ Hamburger Sternwarte, Universität Hamburg, Gojenbergsweg 112, 21029 Hamburg, Germany \\ 2 Chandra X-ray Center, Harvard-Smithsonian Center for Astrophysics, 60 Garden Street, Cambridge, MA 02138, USA \\ 3 Max-Planck-Institut für extraterrestrische Physik (MPE), Postfach 1312, 85741 Garching, Germany
}

Received 24 November 2003 / Accepted 7 January 2004

\begin{abstract}
We report the first unambiguous detection of X-ray emission originating from Saturn with a Chandra observation, duration $65.5 \mathrm{ks}$ with ACIS-S3. Beyond the pure detection we analyze the spatial distribution of X-rays on the planetary surface, the light curve, and some spectral properties. The detection is based on 162 cts extracted from the ACIS-S3 chip within the optical disk of Saturn. We found no evidence for smaller or larger angular extent. The expected background level is $56 \mathrm{cts}$, i.e., the count rate is $(1.6 \pm 0.2) \times 10^{-3} \mathrm{cts} / \mathrm{s}$. The extracted photons are rather concentrated towards the equator of the apparent disk, while both polar caps have a relative photon deficit. The inclination angle of Saturn during the observation was $\sim-27^{\circ}$, so that the northern hemisphere was not visible during the complete observation. In addition, it was occulted by the ring system. We found a small but significant photon excess at one edge of the ring system. The light curve shows a small dip twice at identical phases, but rotational modulation cannot be claimed at a significant level. Spectral modeling results in a number of statistically, but not necessarily physically, acceptable models. The X-ray flux level we calculate from the bestfit spectral models is $\sim 6.8 \times 10^{-15} \mathrm{erg} \mathrm{cm}^{-2} \mathrm{~s}^{-1}$ (in the energy interval $0.1-2 \mathrm{keV}$ ), which corresponds to an X-ray luminosity of $\sim 8.7 \times 10^{14} \mathrm{erg} \mathrm{s}^{-1}$. A combination of scatter processes of solar X-rays require a relatively high albedo favoring internal processes, but a definitive explanation remains an open issue.
\end{abstract}

Key words. planets and satellites: general - planets and satellites: individual: Saturn - X-rays: general

\section{Introduction}

X-ray emission from solar system objects has so far been detected from the Earth (Grader et al. 1968; Rugge et al. 1979; Fink et al. 1988), from the Moon (Gorenstein et al. 1974; Schmitt et al. 1991), from a number of comets (e.g., Lisse et al. 1996; Dennerl et al. 1997; Mumma et al. 1997), from Jupiter (Metzger et al. 1983), from the Galilean satellites Io and Europa (Elsner et al. 2002), from Venus (Dennerl et al. 2002), Mars (Dennerl 2002), and marginally from Saturn (Ness $\&$ Schmitt 2000). The observed X-ray emission appears to have different physical origins in the different objects. The principal X-ray production mechanism for Moon, Earth, Venus, and Mars is scattering of solar X-rays. Auroral X-ray emission has been found from the Earth and from Jupiter, and similar emission from the outer planets is anticipated.

Aurorae on Earth and Jupiter are generated by charged particles precipitating into the planetary atmosphere along the magnetic field lines. While at Earth the precipitating flux consists of solar wind electrons, Einstein observations were interpreted to show that the Jovian X-rays are caused by heavy

Send offprint requests to: J.-U. Ness, e-mail: jness@hs.uni-hamburg.de ion precipitation with oxygen and sulfur ions originating from the volcanically active moon Io (e.g., Metzger et al. 1983). Further support for this scenario came from a direct observation of heavy ions in Jupiter's magnetosphere with the Voyager spacecraft and from a comparison of ROSAT observations in the soft X-ray spectrum with model-generated bremsstrahlung and line emission spectra (Waite et al. 1994). In an analysis of $\mathrm{O}_{\text {I }}$ ( 21304) and S II (21256) measured with HST, Trafton et al. (1998) found only upper limits, but they note that these upper limits were still consistent with the existence of sufficient heavy ions among the precipitating particles to explain the X-ray observations. However, a recent Chandra HRC observation of Jupiter carried out in December 2000 for an entire $10 \mathrm{~h}$ rotation (Waite 2002; Gladstone \& Majeed 2001) has put serious doubt on this theory. The X-ray emission in their highresolution image is found to be concentrated near the magnetic poles, and a peculiar 45 min pulsation similar to high-latitude radio pulsations previously detected by the Galileo and Cassini spacecraft was found. The production of X-ray emission concentrated so close to the poles cannot be explained by ions coming from near Io's orbit. The polar emission was identified to be stronger at the north pole than in the south polar region (Waite et al. 1996). Also, equatorial emission was identified at 
a low level, probably originating from different scatter mechanisms of solar X-rays. An analysis of Chandra observations with ACIS from November 1999 also showed evidence for soft $\mathrm{X}$-ray emission from the Galilean satellites Io and Europa, and probably Ganymede (Elsner et al. 2002). They interpret the emission as a result of bombardment of their surfaces by energetic $(>10 \mathrm{keV}) \mathrm{H}, \mathrm{O}$, and $\mathrm{S}$ ions originating from the region of the Io Plasma Torus (IPT). The IPT itself was found to emit soft X-rays, which appears at the low end of the ACIS-S3 energy band, but Elsner et al. (2002) found an unresolved line or line complex indicative of oxygen.

\subsection{Previous $X$-ray observations of Saturn}

Saturn was observed with the Einstein Observatory IPC for about $10 \mathrm{ks}$, but no X-ray emission was detected, leading Gilman et al. (1986) to the conclusion that instead of heavy ion precipitation, electron bremsstrahlung was the more likely $\mathrm{X}$-ray production mechanism for Saturn. With this assumption they calculated a $3 \sigma$ upper limit for the Saturnian X-ray flux at Earth of $1.7 \times 10^{-13} \mathrm{erg} \mathrm{cm}^{-2} \mathrm{~s}^{-1}$, a value consistent with an expected energy flux at Earth of $8 \times 10^{-16} \mathrm{erg} \mathrm{cm}^{-2} \mathrm{~s}^{-1}$, obtained from a model calculation by Gilman et al. (1986) based on UV observations (Sandel et al. 1982) and the assumption of thick-target bremsstrahlung at high latitudes.

A marginal X-ray detection of Saturn was obtained in a systematic analysis of ROSAT PSPC data on trans-Jovian planets by Ness \& Schmitt (2000), although no detection was expected from auroral thick target bremsstrahlung models. In a $5349 \mathrm{~s}$ PSPC observation 22 counts were recorded in a box centered at the position of Saturn while only 7.6 counts were expected from background. The probability of measuring 22 counts or more with only 7.6 counts being expected is $1.7 \times 10^{-5}$, assuming Poisson statistics, hence the formal significance of the detection is quite high. The 14.4 counts, formally attributed to Saturn correspond to an energy flux of $1.9 \times 10^{-14} \mathrm{erg} \mathrm{cm}^{-2} \mathrm{~s}^{-1}$ using a conversion factor of $6 \times 10^{-12} \mathrm{erg} \mathrm{cm}^{-2} \mathrm{~s}^{-1}$. This value is significantly higher than the model calculation by Gilman et al. (1986) but it is not in contradiction to the upper limit estimated from the Einstein observation. In any case, Saturn is not as X-ray bright as Jupiter, and little could be inferred about the X-ray spectrum of Saturn, except that it should be very soft since no detection in the hard ROSAT band was obtained. Also, nothing could be derived about the spatial location of the Saturnian X-ray source because of the low counting statistics and the low angular resolution of the ROSAT PSPC. A recent observation of Saturn with XMM-Newton is presented by Ness et al. (2004) and their results compare very well with our Chandra observations.

We present a new observation of Saturn carried out with Chandra. With the high spatial resolution of the ACIS detectors, we choose an exposure time of $\sim 70 \mathrm{ks}$, sufficiently high to detect X-ray emission even if, in a worst case, the emission was randomly distributed over the planetary surface. The observation setup and data analysis is described in Sect. 2, the results are described in Sect. 3 and discussed in Sect. 4, and our conclusions are presented in Sect. 5.
Table 1. Overvation details for Saturn.

\begin{tabular}{lr}
\hline \hline ObsID & $3725 / 4433$ \\
Exp. time & $71.5 \mathrm{ks}$ \\
On-time & $65.5 \mathrm{ks}$ \\
Start time & $2003-04-1407: 53$ \\
Stop time & $2003-04-1504: 17$ \\
$\mathrm{RA}^{a}$ & $05^{\mathrm{h}} 35^{\mathrm{m}} 46^{\mathrm{s}}-05^{\mathrm{h}} 36^{\mathrm{m}} 06^{\mathrm{s}}$ \\
Dec $^{a}$ & $22^{\circ} 21^{\prime} 57^{\prime \prime}-22^{\circ} 22^{\prime} 30^{\prime \prime}$ \\
Angular diam. & $17.5^{\prime \prime}$ \\
Distance (Earth) & $9.5 \mathrm{AU}$ \\
Distance (Sun) & $9.0 \mathrm{AU}$ \\
Inclination & $-27^{\circ}$ \\
\hline
\end{tabular}

\section{Observations and data analysis}

\subsection{Observations}

In April 2003, we obtained an X-ray observation of Saturn with ACIS-S3 aboard Chandra for a total of $71.5 \mathrm{ks}$, but Saturn was actually only $65.5 \mathrm{ks}$ in the field of view (see Table 1 ). We used the back-illuminated $\mathrm{S} 3$ chip in order to take advantage of this CCD's sensitivity to low energy X-rays. The primary concern of the observational setup was loading the CCD due to optical light from Saturn. Scaling from earlier observations of Jupiter, we expected an optical load of about 8 ADU/pixel per $3.2 \mathrm{~s}$ ACIS frame. While this is below the event split event definition and would not by itself create false events, it would strongly bias the data toward higher energies. We used the 1/4 subarray mode to reduce the frame time and thus lower the expected optical loading to about $2 \mathrm{ADU} / \mathrm{pixel} /$ frame. In order to measure and correct for the residual loading of the CCD due to optical light from Saturn we used the "Very Faint" (VF) telemetry format. The telemetry saturation limit in this mode is 68.8 events per second. We found on average 5 events per second and clearly lost no frames. The spacecraft was re-pointed after the first $35 \mathrm{ks}$ to follow the planet's proper motion. Each pointing was oriented so that the planetary motion went from one extrema to the other of the subarray traveling along the CCD's node one, perpendicular to the long axis of the subarray.

The observation setup was chosen to prevent optical loading. From the photon events (Fig. 1) no indication for the rings can be recognized, which would have shown up in the case of optical loading. We therefore conclude that the setup was successful. Nevertheless we applied an energy correction scheme "biasevt1.pl" developed by Peter G. Ford from MIT. This perl script corrects the nominal $3 \times 3$ event island by using information contained in the $5 \times 5$-pixel event island telemetered in the Very Faint Mode. The typical region of influence of an X-ray event is limited to a central pixel and adjacent pixels. Members of the event island separated from the central pixel by an intermediate pixel provide information on the local background. For each event, biasevt1.pl subtracts the mean PHA value calculated from the outer 16 pixels from the PHA of each of the inner nine pixels. 
Filters are applied to reject background which is contaminated by an X-ray event. This simulates what the PHA values would have been in the absence of optical loading. The mean shift per pixel in the $3 \times 3$ event island was 1.14 ADU, consistent with expectations. The data are then reprocessed with the CIAO tool acis_process_events to recalculate the event's true total pulse height after Charge Transfer Inefficiency (CTI) corrections are made. Finally acis_process_events applied a gain correction to each event to determine the final energy. The pixel coordinates listed in the processed data file are converted into sky-centered RA and Dec positions using the CIAO dmcopy command. In order to test for the effects from the energy correction scheme, we compared the spectra obtained from the corrected dataset and from the non-corrected dataset. We found the two spectra to be practically identical as expected from the small ( $<2$ ADU) spectral shift.

\subsection{How to find Saturn's X-ray photons}

The photon positions on the original sky-centered image (in RA/Dec coordinates) show no trace of Saturn nor can any strong background sources be identified. We calculated an expected path using ephemeris data from the JPL/SSD ephemeris generator $^{1}$ and orbit data of the satellite provided in the orbit file (which is part of the ephemeris products delivered with the observation). The coordinates of Saturn at start and stop time of the observation are given in Table 1 .

From the expected position of Saturn at any given time during the observation we calculate offset coordinates with respect to the center of Saturn from the RA and Dec coordinates for each individual photon ${ }^{2}$. This transformation shifts all photons that potentially originate from Saturn to a central "saturnocentric" position. We verified our extraction procedures with the Chandra data on Jupiter, which is so strong, that all $\mathrm{X}$-ray photons originating from Jupiter can be identified without any shifting. In Fig. 1 the transformed photon positions are shown in "saturnocentric" coordinates. The extraction regions for the source (circle with 17.5" diameter at origin; see Table 1) and background (large boxes above and below the source) are overlaid. The effective exposure time is significantly smaller towards high offset values, an effect due to the transformation. We investigated this effect by constructing a rough exposure map consisting of nine vertical strips. For each strip we calculate the effective exposure time from the difference of photon arrival times of the first and last photon. We found the effective exposure time to vary significantly from the central strip to high offset values. For the background extraction we therefore choose the extraction regions near the RA position of the source and the offset mainly in Dec direction, i.e., above and below the source. The extraction regions are marked in Fig. 1 and the number of photons counted in each extraction region is given above the respective region.

\footnotetext{
${ }^{1}$ http://ssd.jpl.nasa.gov/cgi-bin/eph

2 Since the RA axis increases from right to left we flipped the offset positions for a correct representation, yielding up $=$ North and left $=$ East.
}

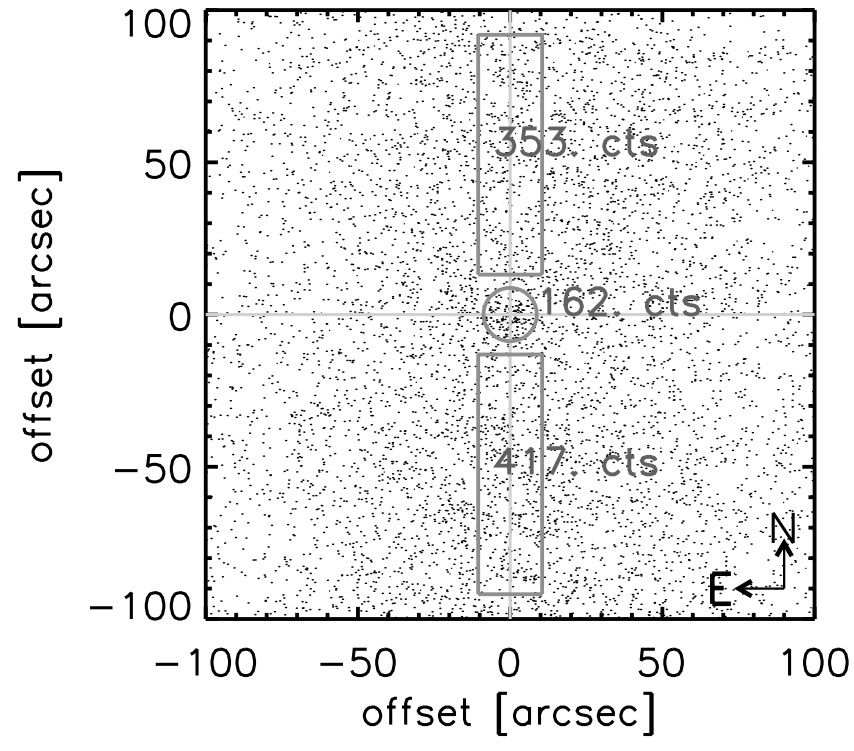

Fig. 1. Photon events of Saturn extracted for $E=0.1-2 \mathrm{keV}$. The source photons are extracted from a circular region with the apparent diameter of Saturn (17.5", thus an extraction area of $\left.240.528^{\prime \prime 2}\right)$ and the background photons from within two $21^{\prime \prime} \times 78.75^{\prime \prime}$ boxes above and below the source (total area 3307.5 ${ }^{\prime 2}$ ). Plotted are the photon shifts relative to Saturn.

With the chosen background method we neglect effects from background contributions from real X-rays from behind Saturn, which will show up in our extracted background, but are blocked by the planet in the source extraction region. This implies an overestimation of the instrumental background and thus an underestimation of the source flux. Background studies at high galactic latitudes were carried out by, e.g., Markevitch et al. (2003), indicating a low X-ray background contribution at sufficiently high galactic latitudes. From their wide-band fluxes we estimate the source flux for Saturn to be underestimated by at most $10 \%$. For conservative analysis we apply no corrections to our instrumental background.

\subsection{Lightcurve and spectrum}

For the analysis of the X-ray lightcurve and the X-ray spectrum of Saturn the photons within a circle of $10^{\prime \prime}$ radius around its nominal position were used. This radius is somewhat larger than the $8.75^{\prime \prime}$ radius of the Saturnian disk, to ensure that all photons from Saturn are collected, allowing for uncertainties in the absolute attitude reconstruction and some redistribution of the photons due to the PSF of the X-ray telescope. In order to avoid any effect from different exposure times along the $x$-axis, we choose the extraction regions for the background as vertical strips above and below the source, just as in Fig. 1. With this extraction radius we analyze 197 counts with an expectation of 72.4 counts from the background. We binned the arrival times and the photon energies with binsizes $4.6 \mathrm{ks}$ for the light curve and $50 \mathrm{eV}$ energy bins for the spectrum. For the spectrum we used only photons below $2 \mathrm{keV}$ (Fig. 6). We counted the photons assigned to Saturn and to the background separately (see Fig. 1) in each bin in order to obtain a spectrum 
and a lightcurve for the source and the background. The results are shown in Figs. 5 and 6 and are discussed in Sect. 3.

For the purpose of spectral modeling with XSPEC the raw spectrum of Saturn was adaptively rebinned so that each bin contains at least 15 photons. The spectral modification due to the contamination layer on ACIS was taken into account by a multiplicative term acisabs.

\section{Results}

\subsection{Identification of $X$-rays from Saturn}

In Fig. 1 we show the result from our transformation procedure. The axes give the offset coordinates from the center of Saturn. From the circular extraction region centered on Saturn's expected position we find 162 counts in the energy range $0.1-2 \mathrm{keV}$ within the known optical extent of Saturn's disk. We estimate the number of background photons (assuming of course a constant background level) contained in the source extraction region to be 56 counts. Obviously, this detection is highly significant. The probability of measuring 162 counts with 56 expected from the background is zero for all practical purposes. Therefore the ROSAT detection of Saturn reported by Ness \& Schmitt (2000) is confirmed. With these numbers and the assumption of Poissonian statistics we calculate a net count rate of $(106 \pm 12.7 \mathrm{cts}) / 65.5 \mathrm{ks}=$ $(1.6 \pm 0.2) \times 10^{-3} \mathrm{cts} / \mathrm{s}$.

\subsection{Spatial distribution of Saturnian X-ray emission}

In order to identify Saturn as an X-ray source in our Chandra image we assumed a spatial extent identical to the apparent optical diameter of Saturn's disk. Having found X-ray emission from Saturn we calculated the signal-to-noise ratio of the signal attributable to Saturn as a function of the radius of the chosen annular extraction region. We find a relatively broad maximum extending about 1" beyond Saturn's apparent optical radius, however, given the relatively small number statistics and the broad nature of the maximum, we conclude that there is at the moment no reason to assume an X-ray halo extending substantially above Saturn's limb.

We next investigated the spatial distribution of the recorded photons on Saturn's apparent disk. In Fig. 2 we plot the photons over an optical image ${ }^{3}$ taken simultaneously with the Chandra observation. At the time of our observation the Saturnian rings covered the northern hemisphere potentially blocking X-ray emission originating from the northern polar region. The planet was inclined by $-27^{\circ}$. The geometrical equator is marked by a red line and the symbols representing the recorded X-ray photons are scaled with the respective photon energies. We identify a concentration of $500 \mathrm{eV}$ and $800 \mathrm{eV}$ photons on Saturn's disk compared to the background. In order to investigate the homogeneity of the X-ray emission we divided Saturn's apparent disk into three regions, a northern cap (NC) and southern (SC) polar cap with each $28.7 \%$ of the total area, and an equatorial belt (EB) containing $42.6 \%$ of the total area. Assuming

\footnotetext{
${ }^{3}$ Provided by Bernd Flach-Wilken, April 14, 19h UT, with 300mmSchiefspiegler Feff $=6 \mathrm{~m}$, ST237, $18 \times 0.1 \mathrm{~s}+$ Philips ToUCam, $20 \mathrm{~s}$.
}

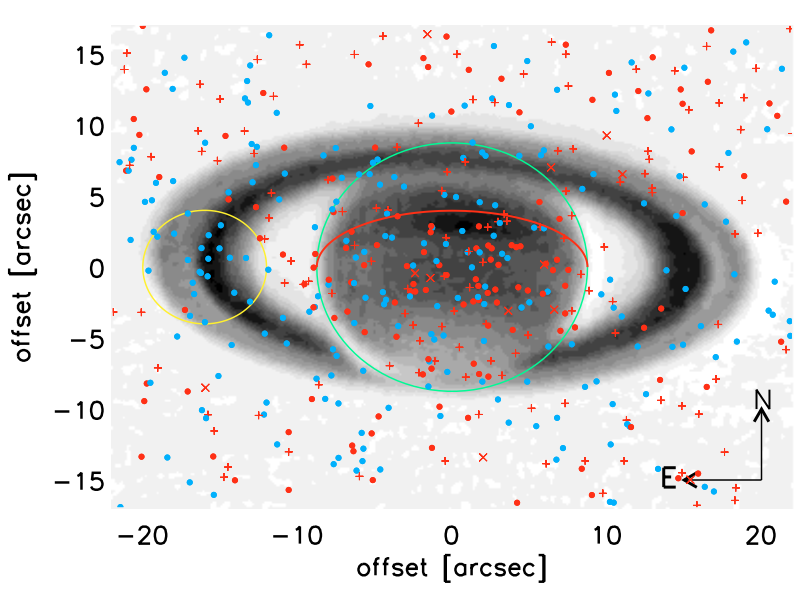

Fig. 2. Optical image of Saturn ${ }^{3}$ at the time of the Chandra observation with the $\mathrm{X}$-ray photons overlaid. The large circle marks the extraction region with diameter $17.5^{\prime \prime}$, the smaller circle indicates an excess at the edge of the ring system. The planet was inclined by $-27^{\circ}$ at the time of the observation. Symbols mark photon energies: $<200 \mathrm{eV}(\times), 200-1000 \mathrm{eV}(\bullet)$, and $>1000 \mathrm{eV}(+)$. Photons with energies between 200 and $700 \mathrm{eV}$ are plotted with lighter colors.

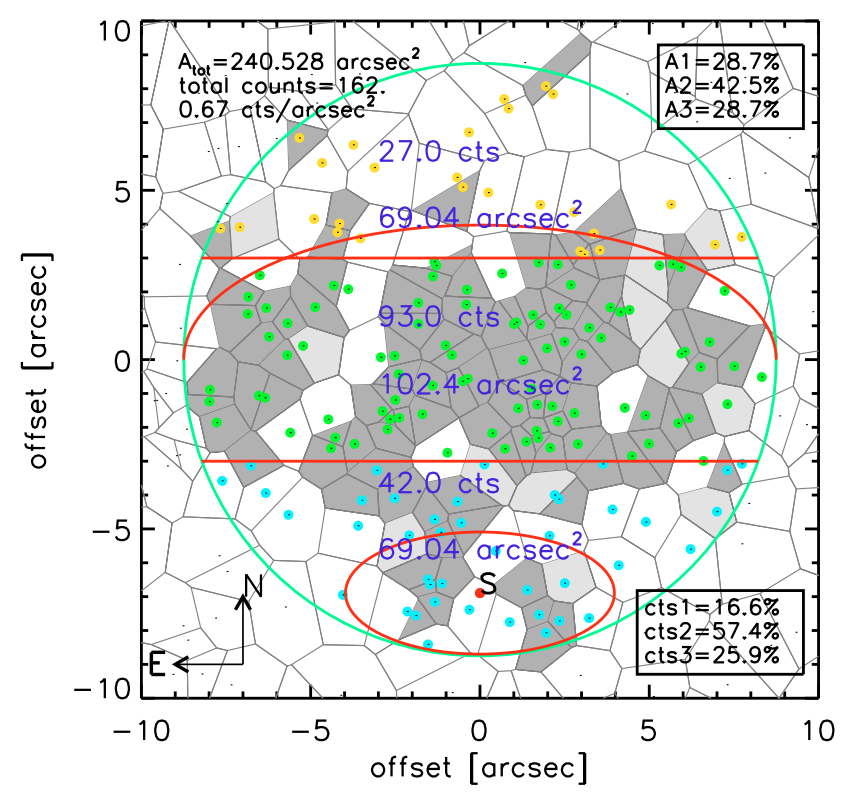

Fig. 3. Spatial distribution of X-ray photons within the source extraction region. The polygons are Voronoi regions comprising all points closer to each particular photon. The 106 smallest areas are grey shaded indicating a statistical approach to identify the net photons. Twelve more areas are shaded with lighter grey to indicate the $1 \sigma$ tolerance. Three areas, NC, EB, and SC (see text) are defined to associate polar and equatorial photons. The geometrical equator and the visible part of the south polar region are given by the red lines $\left(i=-27^{\circ}\right)$.

an equal surface brightness disk we would expect 46.5, 69.0, and 46.5 counts in NC, EB, and SC, respectively, which has to be contrasted with 27,93 , and 42 counts actually recorded in $\mathrm{NC}, \mathrm{EB}$, and $\mathrm{SC}$, respectively. The procedure is illustrated in Fig. 3. We carried out the same analysis with the much higher SNR data available for Jupiter and found a concentration in the polar caps compared to the equatorial belt. Obviously, the spatial distribution of the X-ray emission in Jupiter and Saturn is 
quite different. Saturn's X-ray emission is indeed distributed inhomogeneously over its apparent disk. While for Jupiter there is a concentration towards the poles, there is a deficit of emission from Saturn's northern polar region, which was occulted by the ring system at the time of our observations. However, the southern polar cap shows a deficit as well and there is definitely a detectable concentration of the X-ray emission towards the equator of the apparent disk. Testing the hypothesis of a uniform distribution over the disk excluding the northern cap we find an expected total number of uniformly distributed counts in $\mathrm{EB}$ and SC of 80.6 counts in EB and 54.4 counts in SC, which has to be contrasted with 93 and 42 counts, respectively. Under the assumption of Poissonian statistics we calculate a total (reduced) $\chi_{\text {red }}^{2}=2.64$. This value allows the hypothesis of a uniform distribution to be true with a probability of less than $10 \%\left(\chi_{\text {red }}^{2}=2.99\right)$ but more than $5 \%\left(\chi_{\text {red }}^{2}=2.3\right)$.

For a visual impression we constructed Voronoi areas comprising all points closer to each particular photon. Since from the 162 photons counted within the optical radius, 56 are statistically expected to belong to the background, we mark the 106 smallest Voronoi polygons with grey color in Fig. 3 in order to identify regions of high source intensity. The next 12 smallest areas are marked with a lighter grey to mark a $1 \sigma$ confidence level. It can be seen that the highest concentration is found in the center of the image. With the inclination angle of $-27^{\circ}$ all emission therefore originates from the southern hemisphere, but an enhancement of source emission towards the south pole cannot be identified. This effect is difficult to understand in terms of internal production mechanisms, but some scattering processes of solar X-rays (backscattering, fluorescent scattering) could be an explanation for the geometrical distribution of X-rays, if we assume that the rings have a much lower X-ray albedo than the planetary disk and that they attenuate any X-ray radiation from the planet below.

From close inspection of the individual X-ray photons overlayed over the optical image in Fig. 2 we identify some excess in X-rays coinciding with one edge of the ring system which cannot be seen on the other side of the rings. We marked this area by the smaller circle at $x \sim-16^{\prime \prime}$ and it is remarkable that the photons in this region have all roughly the same energy. In the same fashion as for Saturn (Sect. 2.2 and Fig. 1) we extract those photons within a circle of radius 4 " (and background from boxes above and below) and found 22 cts with 11 expected from the background. When doing the same extraction procedure only for photons within $200-900 \mathrm{eV}$, the significance is higher: still $22 \mathrm{cts}$ but with only 6 cts expected from the background. Interestingly an extraction in the $10 \mathrm{ks}$ time interval $36 \mathrm{ks}$ to $46 \mathrm{ks}$ (thus right after the re-pointing) returns $10 \mathrm{cts}$ with only $1.5 \mathrm{cts}$ expected from the background. We checked the arrival times of these photons, but find a concentration at the time right after the re-pointing of the telescope at $t \sim 35 \mathrm{ks}$ not significant. We are not aware of any consequences from the re-pointing that might lead to such a photon excess. From the statistical point of view the detection of $22 \mathrm{cts}$ is significant, at least within the reduced energy interval $200-900 \mathrm{eV}$. We analyzed the original (non-transformed) chip in this energy range and searched for regions with 22 photons or more counted in circles with 4 " radius. The highest count number we found

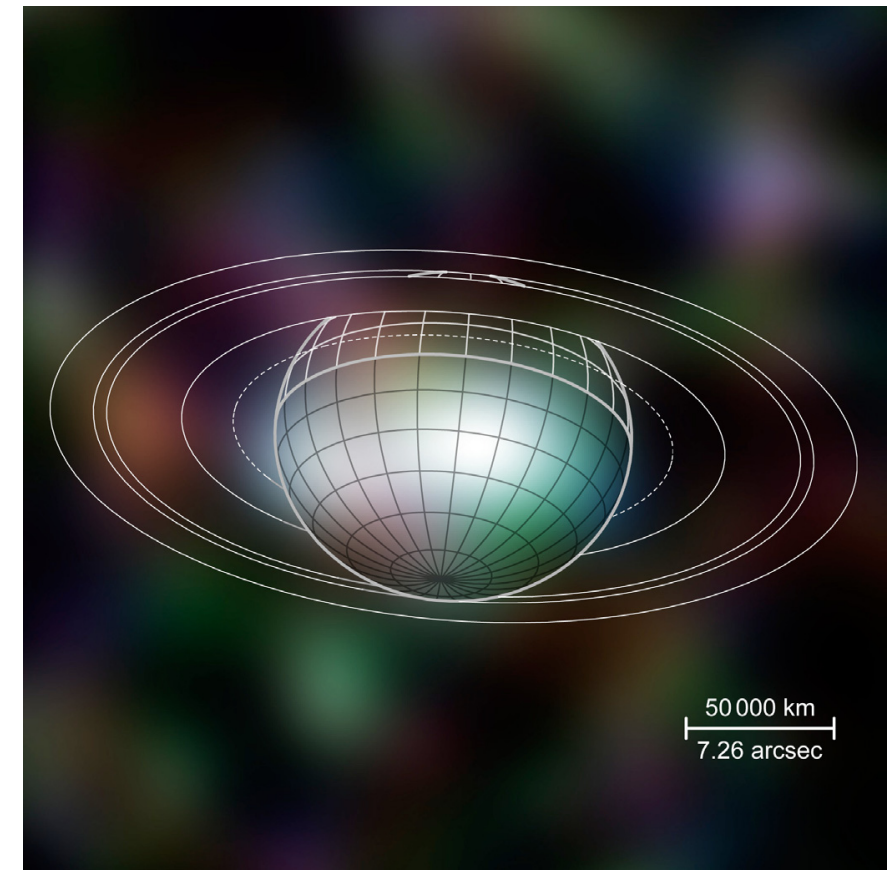

Fig. 4. X-ray colour LRGB image of Saturn in the energy ranges $\quad 0.4-0.6 \mathrm{keV} \quad$ (red), $\quad 0.6-0.8 \mathrm{keV} \quad$ (green), $\quad 0.8-1.0 \mathrm{keV}$ (blue), smoothed using a Gaussian with a $F W H M$ of 5 arcsec. A drawing of Saturn at the time of the observation, obtained from http://ringmaster. arc.nasa.gov/tools/viewer2_satc.html was overlaid for clarity.

was 20 counts, but the count statistics suggests a Poissonian statistics with a peak at only 3.2 counts. With the same search repeated for the chip in transformed coordinates we detected the 22 counts at the edge of the ring system and only one further denser region with 20 counts at $x=12.5^{\prime \prime}$ and $y=-70^{\prime \prime}$. After this exercise we regard the photon excess at the edge of the ring system as significant, but we have no suggestion for a production mechanism. It is not clear why this excess is seen on only one side of the ring system. We checked the moons, but none was near that particular position at any time during the observation ${ }^{4}$. Also, a background source is improbable, because we did not find the source on the non-transformed detector coordinate system. The "light curve" of these photons shows no significant anomaly, such that no instrumental effect can be held responsible.

\subsection{Lightcurve}

As described in Sect. 2 we extracted the lightcurve and the spectrum of the photons within $10^{\prime \prime}$ around the apparent position of Saturn. We plot the lightcurve with a time binning of $4600 \mathrm{~s}$ in Fig. 5. The dip at $35 \mathrm{ks}$ is due to the gap between the two consecutive observations with the Chandra spacecraft being repointed to follow Saturn's apparent motion. For about $6 \mathrm{ks}$ Saturn was actually outside the field of view. The count statistics is obviously poor, however, there is a hint for two dips possibly caused by rotational modulation.

\footnotetext{
4 http://ringmaster.arc.nasa.gov
} 

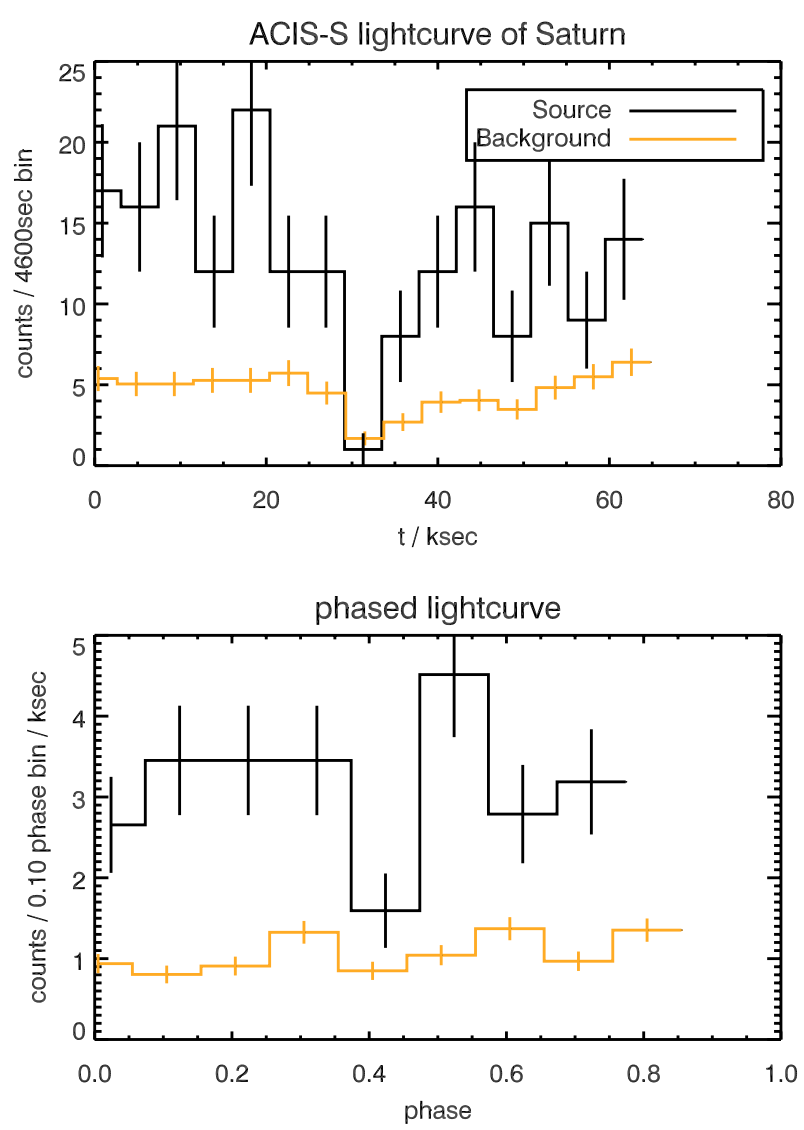

Fig. 5. Light curve with time bins $4600 \mathrm{~s}$ (upper panel) and phased lightcurve obtained from Saturn's rotation period $37.67 \mathrm{ks}$ (=0.436 days; bottom panel) for source and background separately.

We therefore generated a phased lightcurve using the known rotation period of Saturn $(0.436$ days $=37.67 \mathrm{ks})$ shown in the bottom panel of Fig. 5; for Fig. 5 a phase binsize of 0.1 was used; note that because of the exposure history and the rotation period of Saturn almost no coverage was obtained for the phase bin between 0.8 and 1.0. In the phased up light curve there appears to be a minimum between phases $0.35-0.50$. In order to assess the statistical significance of this dip we generated random phased light curves with the same number of photons as recorded from our Saturn observation, uniformly distributed over the available phase space. We determined the number of photons recorded in a phase interval $\Delta \phi=0-0.15$ such that the actual number of recorded photons is minimal. A comparison of those numerical experiments with the numbers obtained for Saturn shows that there is a chance of about $15 \%$ to obtain a phase dip of the same strength as recorded for Saturn in a data set with constant count rate. We therefore conclude that there is no evidence for a rotationally modulated signal and data covering far more than two rotation periods are required for statistically significant studies.

\subsection{Spectrum}

The spectrum of the photons centered on Saturn's position is shown in Fig. 6. The emission feature at $\sim 300 \mathrm{eV}$ can be identified both in the source and in the background. The drop in
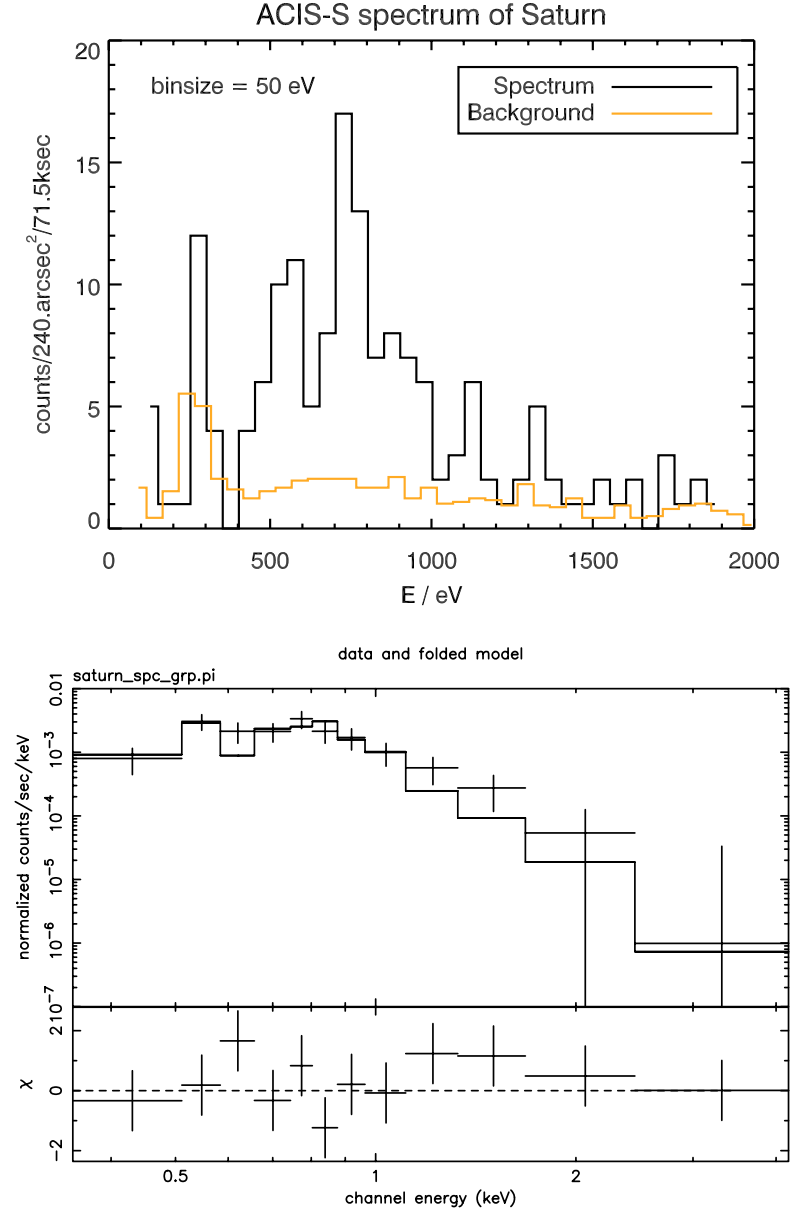

Fig. 6. Extracted spectra for the background and source+background (top). Bottom: best-fit obtained with XSPEC. The model consists of a MEKAL $(k T=0.39 \pm 0.08 \mathrm{keV}$ and solar abundances) and a single line at $0.527 \mathrm{keV}$, only instrumentally broadened.

counts just above $300 \mathrm{eV}$ corresponds to the carbon K-edge in the optical blocking filter. The drop in counts at lower energies is also due to absorption in this filter. Additional emission features between $500 \mathrm{eV}$ and $800 \mathrm{eV}$ can only be identified for the source. We tested a number of spectral models, and in the bottom panel of Fig. 6 we show the rebinned spectrum with our best-fit model, but we point out that the number of photons is insufficient to arrive at firm statements from the spectral modeling alone.

As a first approach we tested models assuming several single production mechanisms (fluorescent scattering, solar wind charge exchange as observed in comets, a powerlaw spectrum, thermal bremsstrahlung, black body, and a MEKAL and Raymond-Smith spectrum, representing continuum plus line emission in thermal equilibrium). We found no convincing proof for any of these models to fully explain the X-ray emission from Saturn. Good fits were found with the black body model $\left(k T=0.18 \mathrm{keV}, \chi_{\text {red }}^{2}=0.7\right.$ for 10 d.o.f. $)$, but it has no physical meaning. MEKAL and Raymond-Smith models yield only good fits with adjusted elemental abundances. However, the spectral resolution does not allow to claim abundance anomalies with high significance. If solar X-ray emission 
was involved, we would expect to see the incident solar X-ray spectrum with solar abundances.

In addition to single production mechanisms we considered a combination of two mechanisms and obtained a good fit with a MEKAL model with solar abundances combined with a single line from oxygen $\mathrm{K}-\alpha\left(\chi_{\text {red }}^{2}=0.9\right.$ for 9 d.o.f.). This fit is shown in Fig. 6. According to the best-fit we find fluxes $(0.1-2 \mathrm{keV})$ of $1.26 \times 10^{-15} \mathrm{erg} \mathrm{cm}^{-2} \mathrm{~s}^{-1}$ to be contained in the fluorescence line and $5.5 \times 10^{-15} \mathrm{erg} \mathrm{cm}^{-2} \mathrm{~s}^{-1}$ in the MEKAL model. The total flux in the energy interval $0.1-2 \mathrm{keV}$ derived from the best fit is $6.7 \times 10^{-15} \mathrm{erg} \mathrm{cm}^{-2} \mathrm{~s}^{-1}$, which is about $35 \%$ of the flux reported by Ness \& Schmitt (2000).

\section{Discussion}

With our $65.5 \mathrm{ks}$ Chandra observation we clearly detect X-ray emission originating from Saturn. The high spatial resolution allows to resolve the spatial origin of the X-rays, the long exposure time covering two rotational periods allows to find temporal anomalies, and the energy resolution allows to extract a spectrum, but all analyses going beyond the pure detection are difficult given the small number of photons. Nevertheless we studied spatial, temporal, and spectral signatures to the extent of qualitative statements, but quantitative analysis requires more photons that can only be gathered with more observing time.

Our spectral modeling with XSPEC cannot give definitive answers for individual production mechanisms. Additional hints come from the spatial distribution and from comparison with other planets. We discuss potential production mechanisms in the following.

\subsection{Single production scenarios}

Auroral X-ray emission is expected to be concentrated towards the polar regions as was found for Jupiter, while for Saturn we found no such concentration in the southern polar region. However, this is required for auroral emission, because Saturn's magnetic field is aligned with the rotation axis. Also, UV auroral emission was found to be concentrated towards Saturn's poles (Trauger et al. 1997). However, a clear deficit of UV emission from the south pole is seen on recent HST observations taken one month before our observations (Karkoschka 2003). The model calculations by Gilman et al. (1986) are based on thick target bremsstrahlung at high latitudes and their flux estimates are significantly below our measured flux level. If the X-ray emission from the south pole reflects the flux level predicted by Gilman et al. (1986), we would not be able to isolate the spectral signature from this emission in the spectrum. In our measurement auroral emission is therefore not detected, but it cannot be excluded that auroral X-ray emission originating only from the northern hemisphere was occulted by the ring system.

Interestingly a Black Body model with $k T=0.18 \mathrm{keV}$ yields a good spectral fit, but a physical meaning is difficult to find. The X-ray flux obtained with this model is $f_{\mathrm{X}}=$ $4.4 \times 10^{-15} \mathrm{erg} \mathrm{cm}^{-2} \mathrm{~s}^{-1}$ in the energy interval $0.1-2 \mathrm{keV}$.
Fluorescent Scattering of solar X-rays in the upper atmosphere of Saturn, the dominant process for the X-ray radiation observed from Venus and Mars, would produce narrow emission lines from the most abundant elements in the atmosphere. Elemental abundances are given by (Cameron 1982): helium $\left(0.14 \mathrm{H}_{2}\right)$, oxygen $\left(1.4 \times 10^{-3} \mathrm{H}_{2}\right)$, carbon $\left(8 \times 10^{-4} \mathrm{H}_{2}\right)$, neon $\left(2 \times 10^{-4} \mathrm{H}_{2}\right)$, and nitrogen $\left(1.8 \times 10^{-4} \mathrm{H}_{2}\right)$. We found spectral models with up to four single (narrow) emission lines discrepant from the spectrum. The oxygen fluorescence flux measured for Mars was $5.4 \times 10^{-5} \mathrm{ph} \mathrm{cm}^{-2} \mathrm{~s}^{-1}$ (Dennerl 2002). With the assumption of equal physical conditions we can scale this flux level to Saturn using the apparent diameter of Mars (20.3") and the distance (1.446 AU). We thus expect fluorescent emission from Saturn a factor 50 lower than for Mars, thus $\sim 10^{-6} \mathrm{ph} \mathrm{cm}^{-2} \mathrm{~s}^{-1}$. When compared to the Blackbody photon flux $\left(8.7 \times 10^{-6} \mathrm{ph} \mathrm{cm}^{-2} \mathrm{~s}^{-1}\right)$ this implies that only about $10 \%$ of the total flux can be caused by fluorescent scattering.

Power Law and Thermal Bremsstrahlung can be excluded from the spectral modeling and the expected spectrum from Solar Wind charge exchange is too soft to explain the measured spectrum.

Backscattering of solar X-rays, as suggestive from the spatial distribution, would result in an X-ray spectrum resembling the incident solar spectrum. Raymond-Smith and MEKAL models yield good fits, but only with adjusted abundances. When assuming the X-ray luminosity of the Sun to be $L_{\mathrm{X}}=2 \times 10^{27} \mathrm{erg} / \mathrm{s}$ (which is a high figure), an albedo for Saturn can be estimated from the measured X-ray flux from Saturn and the distance between the Sun and Saturn (9 AU; Table 1). With the flux obtained from the blackbody model, $f_{\mathrm{X}}=4.4 \times 10^{-15} \mathrm{erg} \mathrm{cm}^{-2} \mathrm{~s}^{-1}$, we find an albedo of $>5.7 \times 10^{-4}$, which appears to be quite high. Schmitt et al. (1991) measured $\mathrm{X}$-ray emission from the Moon $\left(L_{\mathrm{X}}=7.3 \times 10^{11} \mathrm{erg} \mathrm{s}^{-1}\right)$, and they reported clear evidence for scattering of solar X-rays. The albedo of the moon is then calculated to $10^{-5}$. Although elastic backscattering of solar X-rays is suggestive from the spatial distribution, it is not consistent with the solar spectrum, and the albedo would have to be significantly higher than for the moon.

\subsection{Combination of scatter processes}

Since no definitive results were obtained from considering isolated processes, we modeled the spectra of two possible scenarios operating in conjunction with each other. We modeled the spectrum resulting from backscattering (resulting in a MEKAL spectrum with solar abundance) and from fluorescence of the most abundant element besides $\mathrm{H}$ and He: oxygen (narrow emission line at $527 \mathrm{eV}$ ). The combined spectrum yields a good fit with a temperature of $k T=0.39 \pm 0.08 \mathrm{keV}$ in the MEKAL model (Fig. 6, bottom). The formal photon flux in the fluorescence line is only $10 \%$ of the total flux $\left(1.9 \times 10^{-6} \mathrm{ph} \mathrm{cm}^{-2} \mathrm{~s}^{-1}\right)$. This is consistent with the expected fluorescent flux level scaled from Mars, but the remaining $90 \%$ of the emission is yet to be explained.

From the combined spectral model we calculate an X-ray luminosity of $8.7 \times 10^{14} \mathrm{erg} \mathrm{s}^{-1}$. This can be compared to 
Jupiter's equatorial emission reported to be $3.6 \times 10^{15} \mathrm{erg} \mathrm{s}^{-1}$ (Waite et al. 1996), a factor 4.1 higher than Saturn's X-ray luminosity. When scaling for different distance and diameters (Jupiter's distance $5.4 \mathrm{AU}$ and diameter $143000 \mathrm{~km}$ and Saturn's diameter $120500 \mathrm{~km}$ ), we expect a factor 3.9 higher luminosity for Jupiter's equatorial regions, which is consistent with our measured model flux. The similar luminosity levels suggest similar production mechanisms for Jupiter's nonauroral emission and Saturn's total emission. While Waite et al. (1997) explain the equatorial emission by heavy ion precipitation, Maurellis et al. (2000) modeled two alternative mechanisms for low-altitude X-rays and found solar photon scattering ( $90 \%$ elastic scattering and $10 \%$ fluorescent scattering) consistent with ROSAT measurements. However, their models predict non-auroral luminosities $\left(3 \times 10^{7} \mathrm{~W}\right)$ about a factor 10 below the power output derived from the observations $\left(3.6 \times 10^{8} \mathrm{~W}\right.$; Waite et al. 1996). This is in line with our considerations about the required albedo for scatter processes. It would be an interesting finding if the albedo of the gas giants Jupiter and Saturn were so much higher than the albedo for the moon.

\section{Summary and conclusions}

$\mathrm{X}$-ray emission from solar system objects has revealed a large variety of production mechanisms ranging from reflection of solar X-rays (Moon), solar wind charge exchange in comets, fluorescent scattering of solar X-rays in Venus and Mars to magnetically induced auroral emission in Earth and Jupiter. Since Saturn is a gas planet with a magnetic field and auroral UV emission (e.g., Ballester et al. 1985; Trauger et al. 1997; Bhardwaj \& Gladstone 2000), a certain level of X-ray emission has always been expected. We carried out a Chandra observation of Saturn with the intention to unambiguously detect $\mathrm{X}$-ray emission from Saturn. A concentration towards the poles would have been easier to detect, but with our observation settings we were able to establish an unambiguous detection of $\mathrm{X}$-ray emission from Saturn, although no strong spatial concentration of X-ray photons was found. A smoothed image of Saturn with a drawing of Saturn at the time of the observation is shown in Fig. 4. The production mechanism(s) for the detected $\mathrm{X}$-ray emission cannot clearly be allocated from the available data. Possibly a combination of several processes must be considered, but due to the limited spectral signature we focused only on some possible scenarios as isolated cases. We essentially found no convincing case, but the combination of two scatter mechanisms, namely, elastic backscatter and fluorescent scattering of solar X-rays, yield good spectral fits, are consistent with the detected spatial distribution, but require an albedo a factor 50 higher than for the moon.

Clearly, the X-ray production mechanisms for Saturn are different from those for Jupiter. Not only the X-ray flux is significantly lower than Jupiter's flux, but also the spatial distribution appears to be different. A concentration towards the poles as encountered for Jupiter suggests magnetic fields to play an important role, which is the case in most intrinsic X-ray production mechanisms as, e.g., the solar corona. From two ROSAT HRI observations of Jupiter in 1992 and 1994 more auroral X-ray emission from the northern hemisphere than from the south pole was found (Waite et al. 1996). This asymmetry is also seen in UV emission (Livengood 1991). A correlation of auroral activity with infrared emission from $\mathrm{H}^{+}$was reported by Caldwell et al. (1980) for Jupiter and by Stallard et al. (1999) for Saturn with variable infrared fluxes from both poles. From our X-ray observation we have no evidence at all for auroral emission from Saturn, however, the northern polar region was occulted by the ring system. If the north-south asymmetry phenomenon found for Jupiter applies to Saturn as well, more emission with a soft signature is expected to be measured when the north pole is not eclipsed. Such a view was observed by ROSAT, but the detection was so marginal that additional observations with Chandra are necessary to make a better case.

We found our emission level consistent with equatorial emission from Jupiter reported by Waite et al. (1996), and since our X-ray emission is concentrated towards the central part of the apparent disk the same production mechanisms are anticipated. Bhardwaj et al. (2002) point out that elastic scattering $(\sim 90 \%)$ in conjunction with other processes can easily account for Jupiter's non-auroral emission and should not be underestimated. Our spectral models are consistent with this scenario and the spatial distribution is suggestive of scatter processes, but a high albedo is required for scatter scenarios both for Jupiter's equatorial X-ray emission and for the detected X-ray emission from Saturn. We suspect more processes to operate in conjunction with scatter processes producing the high level of X-ray emission at low latitudes. Additional auroral emission at high northern latitudes cannot be ruled out from our observation, but could only be discovered when the northern hemisphere is not eclipsed.

Acknowledgements. J.-U.N. acknowledges support from DLR under 50OR0105.

\section{References}

Ballester, G. E., Feldman, P. D., Moos, H. W., \& Skinner, T. E. 1985, BAAS, 17, 697

Bhardwaj, A., \& Gladstone, G. R. 2000, Adv. Space Res., 26, 1551

Bhardwaj, A., Gladstone, G. R., Elsner, R. F., et al. 2002, in ESA SP-514, Earth-like Planets and Moons, 215

Caldwell, J., Tokunaga, A., \& Gillett, F. C. 1980, BAAS, 12, 692

Cameron, A. G. W. 1982, in Essays in Nuclear Astrophysics, 23

Dennerl, K. 2002, A\&A, 394, 1119

Dennerl, K., Burwitz, V., Englhauser, J., Lisse, C., \& Wolk, S. 2002, A\&A, 386, 319

Dennerl, K., Englhauser, J., \& Truemper, J. 1997, Science, 277, 1625

Elsner, R. F., Gladstone, G. R., Waite, J. H., et al. 2002, ApJ, 572, 1077

Fink, H. H., Schmitt, J. H. M. M., \& Harnden, F. R. 1988, A\&A, 193, 345

Gilman, D. A., Hurley, K. C., Seward, F. D., et al. 1986, ApJ, 300, 453

Gladstone, G. R., \& Majeed, T. 2001, in Two Years of Science with Chandra, Abstracts from the Symposium held in Washington, DC, 5-7 September, 2001

Gorenstein, P., Golub, L., \& Bjorkholm, P. 1974, Moon, 9, 129

Grader, R. J., Hill, R. W., \& Seward, F. D. 1968, JGR, 73, 7149

Karkoschka, E. 2003, in Multi-band observations of Saturn with HST, http://hubblesite.org/newscenter/archive/2003/23/

Lisse, C. M., Dennerl, K., Englhauser, J., et al. 1996, Science, 274, 205 
Livengood, T. A. 1991, Ph.D. Thesis, John Hopkins University, Baltimore, MD

Markevitch, M., Bautz, M. W., Biller, B., et al. 2003, ApJ, 583, 70

Maurellis, A. N., Cravens, T. E., Gladstone, G. R., Waite, J. H., \& Acton, L. W. 2000, Geophys. Res. Lett., 27, 1339

Metzger, A. E., Gilman, D. A., Luthey, J. L., et al. 1983, J. Geophys. Res., 88, 7731

Mumma, M. J., Krasnopolsky, V. A., \& Abbott, M. J. 1997, ApJ, 491, L125

Ness, J.-U., \& Schmitt, J. H. M. M. 2000, A\&A, 355, 394

Ness, J.-U., Schmitt, J. H. M. M., \& Robrade, J. 2004, A\&A, 414, L49

Rugge, H. R., McKenzie, D. L., \& Charles, P. A. 1979, Space Research, XIX, 243

Sandel, B. R., Shemansky, D. E., Broadfoot, A. L., et al. 1982, Science, 215, 548
Schmitt, J. H. M. M., Snowden, S. L., Aschenbach, B., et al. 1991, Nature, 349, 583

Stallard, T., Miller, S., Ballester, G. E., et al. 1999, ApJ, 521, L149

Trafton, L. M., Dols, V., Gérard, J.-C., et al. 1998, ApJ, 507, 955

Trauger, J. T., Stapelfeldt, K. R., Clarke, J. T., Ballester, G. S., \& WFPC2 Science Team. 1997, BAAS, 29, 1256

Waite, J. H. 2002, in The High Energy Universe at Sharp Focus: Chandra Science, ASP Conf. Ser., 262, 19

Waite, J. H., Bagenal, F., Seward, F., et al. 1994, J. Geophys. Res., 99, 14799

Waite, J. H., Gladstone, G. R., Lewis, W. S., et al. 1997, Science, 276, 104

Waite, J. H., Lewis, W. S., Gladstone, G. R., Fabian, A. C., \& Brandt, W. N. 1996, in Roentgenstrahlung from the Universe, 641 